\title{
The step forward for VA ECMO: left ventricular unloading!
}

\author{
Paolo Meani ${ }^{1}$, Federico Pappalardo ${ }^{2}$ \\ ${ }^{1}$ Cardiology Department, Maastricht University Medical Center, Maastricht, The Netherlands; ${ }^{2}$ Department of Cardiothoracic Vascular Anesthesia \\ and Intensive Care, San Raffaele Hospital, Vita Salute University, Milan, Italy \\ Correspondence to: Prof. Federico Pappalardo. Department of Cardiothoracic Vascular Anesthesia and Intensive Care, San Raffaele Hospital, Vita \\ Salute University, Milan, Italy. Email: pappalardo.federico@hsr.it. \\ Provenance: This is an invited Editorial commissioned by Section Editor Dr. Lei Huang (Cardiac Center of Tianjin Third-Central Hospital, Tianjin, \\ China). \\ Comment on: Truby LK, Takeda K, Mauro C, et al. Incidence and Implications of Left Ventricular Distention During Venoarterial Extracorporeal \\ Membrane Oxygenation Support. ASAIOJ 2017;63:257-65.
}

Submitted Sep 05, 2017. Accepted for publication Sep 15, 2017.

doi: $10.21037 /$ jtd.2017.10.14

View this article at: http://dx.doi.org/10.21037/jtd.2017.10.14

VA ECMO is nowadays widely recognized as part of the armamentarium for fighting refractory acute heart failure. However, this system pays immediate gratification with high flows provided and rapid whole-body reperfusion but there is often a price to be paid a day later when the lungs become white. This phenomenon is secondary to the flow changes from the femoral artery which significantly affects the pressure-volume (PV) loop of the left ventricle. Therefore, although the use of VA ECMO is an exciting tool to resuscitate patients dying from acute heart failure, overall survival in this scenario remains poor especially if we target for myocardial recovery of the native heart (1).

In the light of these concepts, the usefulness of VA ECMO is still under debate and its efficacy is variable, depending on numerous factors (2).

After the "resuscitative honeymoon", one of the most important issues occurring with VA ECMO is the result of the retrograde aortic flow, which causes a marked increase in the left ventricular (LV) afterload, impairing myocardial performance especially in severe contractile dysfunction (3). The consequences of this phenomenon are left ventricular distension (LVD) and increase of left ventricular enddiastole pressure (LVEDP), leading to severe pulmonary edema; increased wall stress and myocardial oxygen consumption with global ischemia, jeopardizing the ventricular recovery; stagnation of blood and formation of clots in the heart chambers. This cascade has been well shown in animal models (3) and further affirmed by human studies (4). Despite its clinical relevance, the impact of LVD is poorly understood, even lacking a universal definition to apply for its identification.

Truby et al. from the Columbia University in New York reported a retrospective analysis of their experience with 121 selected VA-ECMO runs, executed from March 2007 to November 2014 in a single center. Regardless of the fact that this study represents a relatively small series, some important points outlined in the paper deserve emphasis.

Firstly, they proposed a novel definition of LVD needing to take into account radiological and hemodynamic parameters within 2 hours of VA ECMO support and dividing their population in three groups. VA ECMO run has been assigned to LVD-if left ventricle distension was not present. The coexistence of pulmonary edema on chest radiograph and pulmonary artery diastolic blood pressure (PADBP) is greater than $25 \mathrm{mmHg}$, described the subclinical LVD group (LVD+). Finally, patients needing immediate decompression following VA ECMO initiation, were included in the clinical LVD group (LVD++). The authors voted in favor of this definition explaining the role of PADBP as surrogate of LVEDP. Moreover, by including two criteria, it was possible to rule out patients suffering from isolated intrinsic pulmonary vascular disorders.

The lack of unique and shared definition poses a pivotal issue. Applying the same criteria and classifying LV distension in the same way, would provide an opportunity to compare easily future studies. On top of 
that, a definition based on objectives parameters obtained from invasive monitoring would not be influenced by the clinician's skills or experience. As a consequence, patients' monitoring should be standardized for VA ECMO. A Swan-Ganz catheter seems to be appropriate and practicable for this purpose, measuring all parameters for defining patients' hemodynamics before and over the course of ECMO support, with the perspective to actively trigger the intervention of $\mathrm{LV}$ unloading if criteria are met. The pulmonary capillary wedge pressure should be considered the best surrogate for estimating the LV end diastolic pressure, as we have to acknowledge that LV dimensions on echocardiography and LVEDP are not strictly correlated in the acute setting of MI $v$ s. decompensated chronic cardiomyopathy, in particular when associated with mechanical or pharmacological inotropic support (5).

Secondly, by being done their definition, the Authors can report the incidence of LVD and its grading in a consecutive series of patients with different etiologies; $35 \%$ of patients have shown some degree of LVD in the first hours after implantation, taking into account those in LVD++ (7\%) and LVD+ groups (22\%). Finally, $16 \%$ of all VA ECMO runs required an intervention to decompress the $\mathrm{LV}$. This data are the benchmark for future work on LVD, as in other experiences the LVD rate fluctuates from $10 \%$ (6) to $68 \%$ (7), with some authors considering "elective" decompression as a possible choice (8). Furthermore, Truby et al. recognized ECPR as a risk factor for decompression, either early and late, hypothesizing that global myocardial stunning might play a crucial role after resuscitation.

Post-cardiac arrest myocardial dysfunction has been well demonstrated in preclinical (9) and clinical studies (10). In a series of 148 patients, undergoing coronary angiography after cardiac arrest, $49 \%$ had myocardial dysfunction presenting elevated LVEDP (10). Despite a non-reduced coronary blood flow in the epicardial arteries during this phase (11), the LV distension might be lead to increased wall stress and myocardial oxygen consumption, worsening the diastolic perfusion and jeopardizing ventricular recovery (12). This global dysfunction is transient, lasting usually 3-5 days, and would be effectively treated by $L V$ unloading $(10,13)$.

Nevertheless, thirdly, the authors reported no difference in survival to discharge among LVD++, LVD+ and LVDgroups, with overall survival of $43 \%$. By dividing patients in those who required left ventricle decompression and those non-decompressed, the event free survival in the first 30 days after ECMO implantation was lower in the decompressed group, considering a composite outcome of death or device transition. Moreover, myocardial recovery was lower in patients requiring left ventricle decompression (decompressed: 5\%, LVD+: 29\%, LVD-: 44\%; P=0.003).

In this scenario, $\mathrm{LV}$ unloading and its specific approaches should assume relevant roles. Different techniques have been developed, which can be surgical, percutaneous or using dedicated devices (2). The authors reported their institutional policy to decompress the LV by percutaneous femoral placement of an Impella 2.5 or CP left ventricular assist device (LVAD; Abiomed, Danvers, MA), which was implanted in 13 out of 19 unloaded VA ECMO runs. This device is a fascinating option, being a catheterbased transaortic axial flow pump, placed percutaneously through a femoral approach and providing the unique possibility to completely or significantly unload the LV. Moazzami et al. presented its effectiveness in LV unloading during ECMO support, improving the hemodynamics, as shown by the reduction in right atrial pressure, pulmonary capillary wedge pressure and LV end-diastolic volume (14). Furthermore, our recent results analyzing patients treated with concomitant VA ECMO and Impella have shown a significantly lower in-hospital mortality and a higher rate of successful bridging to either recovery or next therapy as compared to VA ECMO alone (15).

According to the latter, we strongly believe that unloading the left ventricle is necessary. Furthermore, Impella device might be the better option available. However, applying an unloading technique does not necessarily translate into satisfactory decompression. Again, what does it mean "satisfactory decompression"? Which parameters and which cut-off should we use for monitoring LV unloading? Is the left ventricle decompressed enough? All these questions are still open, needing even future preclinical studies for being clarified.

In conclusion, in the setting of cardiogenic shock the role of residual function and aortic valve opening with pulse pressure have a fundamental role: if the extent of myocardial damage is huge, and eventually further exacerbated by LVD, mechanical unloading is necessary to safely transition the patient to a medium or long term LVAD; if there is some residual function, $\mathrm{LV}$ unloading improves myocardial recovery. In this perspective, we open a new opportunity for active $\mathrm{LV}$ unloading in order to improve the survival rate of this sick patients' population. 


\section{Acknowledgements}

None.

\section{Footnote}

Conflicts of Interest: The authors have no conflicts of interest to declare.

\section{References}

1. Beurtheret S, Mordant P, Paoletti X, et al. Emergency circulatory support in refractory cardiogenic shock patients in remote institutions: a pilot study (the cardiac-RESCUE program). Eur Heart J 2013;34:112-20.

2. Meani P, Gelsomino S, Natour E, et al. Modalities and Effects of Left Ventricle Unloading on Extracorporeal Life support: a Review of the Current Literature. Eur J Heart Fail 2017;19 Suppl 2:84-91.

3. Ostadal P, Mlcek M, Kruger A, et al. Increasing venoarterial extracorporeal membrane oxygenation flow negatively affects left ventricular performance in a porcine model of cardiogenic shock. J Transl Med 2015;13:266.

4. Kawashima D, Gojo S, Nishimura T, et al. Left ventricular mechanical support with Impella provides more ventricular unloading in heart failure than extracorporeal membrane oxygenation. Asaio j 2011;57:169-76.

5. Lancellotti P, Price S, Edvardsen T, et al. The use of echocardiography in acute cardiovascular care: recommendations of the European Association of Cardiovascular Imaging and the Acute Cardiovascular Care Association. Eur Heart J Acute Cardiovasc Care 2015;4:3-5.

6. Schwarz B, Mair P, Margreiter J, et al. Experience with percutaneous venoarterial cardiopulmonary bypass for emergency circulatory support. Crit Care Med 2003;31:758-64.

Cite this article as: Meani P, Pappalardo F. The step forward for VA ECMO: left ventricular unloading! J Thorac Dis 2017;9(11):4149-4151. doi: 10.21037/jtd.2017.10.14
7. Fiser WP, Yetman AT, Gunselman RJ, et al. Pediatric arteriovenous extracorporeal membrane oxygenation (ECMO) as a bridge to cardiac transplantation. J Heart Lung Transplant 2003;22:770-7.

8. Hacking DF, Best D, d'Udekem Y, et al. Elective decompression of the left ventricle in pediatric patients may reduce the duration of venoarterial extracorporeal membrane oxygenation. Artif Organs 2015;39:319-26.

9. Kern KB, Hilwig RW, Berg RA, et al. Postresuscitation left ventricular systolic and diastolic dysfunction. Treatment with dobutamine. Circulation. 1997;95:2610-3.

10. Laurent I, Monchi M, Chiche JD, et al. Reversible myocardial dysfunction in survivors of out-of-hospital cardiac arrest. J Am Coll Cardiol 2002;40:2110-6.

11. Kern KB, Hilwig RW, Rhee KH, et al. Myocardial dysfunction after resuscitation from cardiac arrest: an example of global myocardial stunning. J Am Coll Cardiol 1996;28:232-40.

12. Aissaoui N, Guerot E, Combes A, et al. Two-dimensional strain rate and Doppler tissue myocardial velocities: analysis by echocardiography of hemodynamic and functional changes of the failed left ventricle during different degrees of extracorporeal life support. J Am Soc Echocardiogr 2012;25:632-40.

13. Ruiz-Bailén M, Aguayo de Hoyos E, Ruiz-Navarro S, et al. Reversible myocardial dysfunction after cardiopulmonary resuscitation. Resuscitation 2005;66:175-81.

14. Moazzami K, Dolmatova EV, Cocke TP, et al. Left Ventricular Mechanical Support with the Impella during Extracorporeal Membrane Oxygenation. J Tehran Heart Cent 2017;12:11-4.

15. Pappalardo F, Schulte C, Pieri M, et al. Concomitant implantation of Impella (R) on top of veno-arterial extracorporeal membrane oxygenation may improve survival of patients with cardiogenic shock. Eur J Heart Fail 2017;19:404-12. 